\title{
Evaluation of the Grell-Freitas Convective Scheme in the Hurricane Weather Research and Forecasting (HWRF) Model
}

\author{
Mrinal K. Biswas, ${ }^{\mathrm{a}}$ Jun A. Zhang, ${ }^{\mathrm{b}}$ Evelyn Grell, ${ }^{\mathrm{c}}$ Evan Kalina, ${ }^{\mathrm{d}}$ Kathryn Newman, ${ }^{\mathrm{a}}$ \\ Ligia BERNARDET, ${ }^{\mathrm{e}}$ LAURIE CARSON, ${ }^{\mathrm{a}}$ JAMES Frimel, ${ }^{\mathrm{e}}$ AND GEORG GRELL ${ }^{\mathrm{g}}$

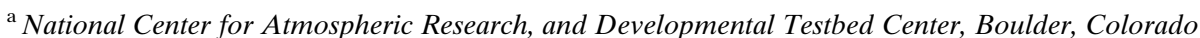 \\ ${ }^{\mathrm{b}}$ NOAA/Atlantic Oceanographic and Meteorological Laboratory/Hurricane Research Division, and Cooperative Institute \\ for Marine and Atmospheric Studies, University of Miami, Miami, Florida \\ ${ }^{\mathrm{c}}$ University of Colorado, Cooperative Institute for Research in Environmental Sciences, NOAA/Earth System Research \\ Laboratory/Physical Sciences Division, and Developmental Testbed Center, Boulder, Colorado \\ ${ }^{\mathrm{d}}$ University of Colorado, Cooperative Institute for Research in Environmental Sciences, NOAA/Earth System Research \\ Laboratory/Global Systems Division, and Developmental Testbed Center, Boulder, Colorado \\ ${ }^{\mathrm{e}}$ NOAA/Earth System Research Laboratory/Global Systems Division, and Developmental Testbed Center, \\ Boulder, Colorado \\ ${ }^{\mathrm{f}}$ Colorado State University, Cooperative Institute for Research in the Atmosphere, NOAA/Earth System Research \\ Laboratory/Global Systems Division, and Developmental Testbed Center, Boulder, Colorado \\ ${ }^{\mathrm{g}}$ NOAA/Earth System Research Laboratory/Global Systems Division, Boulder, Colorado
}

(Manuscript received 18 June 2019, in final form 29 January 2020)

\begin{abstract}
The Developmental Testbed Center (DTC) tested two convective parameterization schemes in the Hurricane Weather Research and Forecasting (HWRF) Model and compared them in terms of performance of forecasting tropical cyclones (TCs). Several TC forecasts were conducted with the scale-aware Simplified Arakawa Schubert (SAS) and Grell-Freitas (GF) convective schemes over the Atlantic basin. For this sample of over 100 cases, the storm track and intensity forecasts were superior for the GF scheme compared to SAS. A case study showed improved storm structure for GF when compared with radar observations. The GF run had increased inflow in the boundary layer, which resulted in higher angular momentum. An angular momentum budget analysis shows that the difference in the contribution of the eddy transport to the total angular momentum tendency is small between the two forecasts. The main difference is in the mean transport term, especially in the boundary layer. The temperature tendencies indicate higher contribution from the microphysics and cumulus heating above the boundary layer in the GF run. A temperature budget analysis indicated that both the temperature advection and diabatic heating were the dominant terms and they were larger near the storm center in the GF run than in the SAS run. The above results support the superior performance of the GF scheme for TC intensity forecast.
\end{abstract}

\section{Introduction}

Hurricane prediction remains a challenging problem for dynamical numerical weather prediction (NWP) and statistical models. It is also challenging to human forecasters who rely on the NWP and statistical model forecasts to issue guidance. On average, global models generally produce the most accurate hurricane track predictions among dynamical models (Cangialosi 2018), but predicting intensity requires high-resolution simulations that are typically performed using limited-area NWP models. The Hurricane Weather Research and

Corresponding author: Mrinal K. Biswas, biswas@ucar.edu
Forecasting (HWRF) model is a limited-area, nestedgrid model that has been operational since 2007. Since then, remarkable improvements in track and intensity forecasts have been achieved, largely due to the initiatives of the Hurricane Forecast Improvement Program (HFIP; Gall et al. 2013). HFIP's 5-yr goal (for 2014) and 10-yr goal (for 2019) are to reduce track and intensity errors by $20 \%$ and $50 \%$, respectively. The reduction in track errors for the HWRF model met the HFIP goals. However, hurricane intensity prediction still remains a challenge. It needs to be mentioned that HWRF is the best deterministic model for intensity prediction in the Atlantic (AL) and east Pacific (EP) basins (Cangialosi 2018; Cangialosi and Franklin 2017). 
As a developing model, HWRF forecasts have improved over the years (e.g., Biswas et al. 2018; see their Figs. 1-3). Table 1 summarizes the major upgrades to the HWRF model starting in 2012. These upgrades include improvements in horizontal and vertical resolution, in data assimilation techniques, in ocean coupling and in physical parameterizations, all of which contributed to the improvements in model performance.

Supported by HFIP, recent upgrades to model physics were transitioned to the operational HWRF model. For instance, improvements in the planetary boundary layer (PBL) scheme were based on observational estimates of the vertical eddy diffusivity of momentum $K_{m}$ made under the developmental physics framework articulated by Zhang et al. (2012). The sensitivity of the tropical cyclone (TC) intensity, track and structure forecasts was tested to understand how the model responded to various $K_{m}$ profiles, particularly those that agreed with observations. This was part of a larger effort to compare the modeled TC structure with observations to identify model deficiencies. Observationally based improvements to the physics were then implemented in the model to reduce biases in the simulated TC structure, which led to improved intensity forecasts. This process was documented by Zhang et al. (2015), which evaluated the impact of the improvement in the HWRF PBL scheme on TC intensity and structure forecasts. Further improvement in the HWRF PBL scheme was documented by Bu et al. (2017) and Wang et al. (2018). The improvement in the horizontal diffusion parameterization of HWRF and its impact on TC forecasts were documented by Zhang et al. (2017). Other physics improvements, such as the inclusion of the partial cloudiness scheme (Biswas et al. 2018) in RRTMG, also contributed to enhanced forecast skill.

Previously, Biswas et al. (2014) showed that track and intensity forecasts using the HWRF model are sensitive to the choice of cumulus parameterization. In addition, multiple modeling experiments have demonstrated the sensitivity of hurricane simulations to the choice of convection scheme (e.g., Shepherd and Walsh 2017; Sun et al. 2014; Pattanayak et al. 2012; Nasrollahi et al. 2012; Smith 2000). For this reason, the convective parameterization in HWRF was identified as a model physics component in which modifications could potentially lead to improvement in TC simulations, particularly in the storm intensity forecast.

In recent years, there has been growing interest in "unified" parameterizations; schemes that are applicable to a wide range of time and space scales, including scales at which the parameterized process may be partially resolved. For convective parameterization schemes (CPSs), which represent the statistical effects of subgrid clouds and their associated vertical motions, a critical underlying assumption of traditional massflux schemes is that the area occupied by active convection is small relative to the size of the model grid box. As the horizontal resolution of NWP models increases into the so-called "grey zone" (grid spacing smaller than approximately $10 \mathrm{~km}$ ), this fundamental assumption may no longer be valid. Arakawa et al. (2011) and Arakawa and Wu (2013) proposed that the impact of the convective parameterization should be dependent on the fraction of the grid box that is assumed to be covered by convection, $\sigma$. As the size of the horizontal grid box decreases and $\sigma$ approaches 1 , the impact of parameterized convection in the grid box decreases as a function of $(1-\sigma)^{2}$, allowing the model to resolve these moist processes explicitly through the microphysical parameterization. The method provides a smooth transition between resolved and unresolved scales.

In a model such as HWRF, scale-independent parameterizations are of particular interest, because the model provides high-resolution coverage of a TC through the use of nested grids of increasing resolution. This means that the physical parameterizations must be valid on the scale of all of the grids on which it is applied. The CPS used in the standard operational version of HWRF, the simplified Arakawa-Schubert (SAS) scheme, was recently modified to include, among other things, the addition of scale "awareness" based on the modulation of the updraft area according to the horizontal grid spacing (Biswas et al. 2018).

The implementation of scale awareness in the SAS scheme largely followed the example of the Grell and Freitas (2014) convective parameterization (hereafter GF), which was the first to successfully apply the scaling ideas of Arakawa et al. (2011) in an operational model [in NOAA's RAPid Refresh (RAP) system (Benjamin et al. 2016)]. The effectiveness of the scaling function in the GF scheme was evaluated by Fowler et al. (2016), using the nonhydrostatic Model for Prediction Across Scales (MPAS), with a variable resolution mesh. They noted that as the horizontal grid spacing became finer, the precipitation physics smoothly transitioned from subgrid to resolved scales.

Due to the demonstrated success of the GF scheme in other models, particularly in an operational application, this scheme was chosen for testing in HWRF. In the work described here, both the SAS and GF schemes are applied in the HWRF system to study the behaviors of these two schemes in simulations of TCs in the Atlantic basin. 


\section{Grell-Freitas and scale-aware SAS schemes}

The SAS and GF schemes have a common ancestry; both can trace their origin to the parameterization developed by Grell (1993), which added downdraft processes to the CPS proposed by Arakawa and Schubert (1974). This scheme was then simplified and widely used in MM5 (Grell et al. 1994), in the early Rapid Update Cycle (RUC) (Benjamin et al. 2004), and in the RAP, which replaced the RUC. The original Grell scheme was further developed by Pan and $\mathrm{Wu}$ (1995) at the National Meteorological Center (now National Center for Environment Prediction) to become the SAS scheme, while a parallel development at NOAA in Boulder led to the Grell and Devenyi (2002; GD) approach. The GF scheme is a development of the GD scheme, which included stochasticism via an ensemble of convective clouds generated by implementing different closure assumptions in the computation of the mass flux. In GF, temporal and spatial correlation patterns can be applied to closure assumptions but also to other internal parameters with large uncertainties. This fully stochastic approach is not used in this study, since no correlation patterns are available for HWRF yet.

Both SAS and GF are mass-flux CPSs, in which the parameterized convective transport is a function of the mass flux through the cloud base. In both schemes, the conceptual framework is the same: a one-dimensional buoyant plume (the cloud model) is modulated by a triggering function, as well as by mixing with environmental air through entrainment, and the environment in turn is modified by the cloud through vertical transport and detrainment. Some features of the SAS scheme are described in Han and Pan (2011), and of the scale-aware SAS in Biswas et al. (2018). For a more complete description of the GF scheme, the reader is referred to Grell and Freitas (2014), Freitas et al. (2018), and Freitas et al. (2020, manuscript submitted to Geosci. Model Dev. Discuss.), with additional background given in Grell (1993) and Grell and Devenyi (2002). Here we focus on some components of particular interest to hurricane applications, as well as some differences between the schemes that may contribute to the differences in the simulations.

In GF, the scale-dependency concept of Arakawa et al. (2011) and Arakawa and Wu (2013) is introduced through both the updraft entrainment rate and through scaling of the cloud base mass flux by $(1-\sigma)^{2}$. First, the convective updraft is assumed to have an initial radius $R$, which is related to the initial entrainment rate $E$, following Simpson et al. (1965) and Simpson (1971), such that $R=0.2 / E$. The fractional coverage of the grid box is expressed as $\sigma=\pi R^{2} / A$, where $A$ is the horizontal area 
of the grid box. The initial entrainment rate is a tuning parameter and is set to $7 \times 10^{-5} \mathrm{~m}^{-1}$. The maximum value of $\sigma$ is restricted (in this case $\sigma_{\max }=0.7$ ), and if $A$ becomes small enough that $\sigma$ would exceed $\sigma_{\max }$, then $\sigma=\sigma_{\max }$ and $R$ is recalculated from $R=\sqrt{(\sigma A / \pi)}$. A new initial entrainment rate is then computed from $0.2 / R$. In this way, for small grid sizes, the entrainment rate increases, which leads to shallower clouds and essentially transitions the scheme to a precipitating shallow convection parameterization. Before being applied to the updrafts, the entrainment rate is adjusted based on the vertical mass flux and on the relative humidity, such that the rate is larger for drier air.

The SAS scheme computes the fractional cloudiness in the same way as the GF scheme; however, the scaling factor is computed at the end of the routine using the actual entrainment rate, rather than an initial assumed rate. Therefore, no adjustment to the entrainment rate based on grid size occurs. The entrainment rate is bounded by the lower limit of $7 \times 10^{-5} \mathrm{~m}^{-1}$ and the upper limit of $3 \times 10^{-4} \mathrm{~m}^{-1}$, and $\sigma$ must be between 0.001 and 0.999 .

Another feature of the schemes that differs is the determination of the mass flux profile of the updraft. In the SAS scheme, the mass flux in and below the cloud is normalized by the mass flux at the cloud base $m_{b}$, modified by entrainment and detrainment. The average vertical velocity and buoyancy of the assumed cloud are the primary factors that determine $m_{b}$. The updraft entrainment rate is the sum of a background turbulent rate and an organized entrainment rate that depends on the relative humidity of the environment outside of the cloud.

In the GF scheme, a probability distribution function (PDF) is used to describe the normalized mass flux, representing a statistical average of deep convective plumes. Using the initial entrainment rate, the PDF is used to calculate entrainment and detrainment rates required to reproduce the normalized vertical mass flux provided by the PDF. An ensemble approach is used in the computation of $m_{b}$, in which multiple realizations of $m_{b}$ are calculated, based on different closure assumptions, to determine the amount and location of convection. To improve efficiency, in the GF implementation, the number of closures is limited to four. These methods of computing $m_{b}$ are based on concepts drawn from multiple CPSs and consider in-cloud stability and buoyancy, vertical motion, integrated vertical advection of moisture, and the time scale for convective adjustment to occur. The mean of the ensemble of $m_{b}$ values is computed and then used to determine the vertical mass flux at a given level.

Both schemes include momentum transport due to convection. The SAS scheme uses a formulation consistent with Zhang and Wu (2003), while GF may use the identical approach as in SAS or a technique from the European Centre for Medium-Range Weather Forecasts (ECMWF) based on Tiedtke (1989). For this work, the ECMWF approach was used. The impact of vertical wind shear on the precipitation efficiency is parameterized in an identical way in the schemes. In addition, both parameterizations include a shallow convection component. In the runs using the GF scheme, the shallow convection was only applied on the outer domain.

The interplay between the convection parameterization and the microphysics parameterization is also important. In the GF scheme, if the relative humidity at the cloud base is greater than $97 \%$ and $\sigma=\sigma_{\max }$, no parameterized convection occurs. It should be noted that for the 2-km grid spacing used on the inner nest of HWRF, $\sigma$ is always equal to $\sigma_{\max }$. Therefore, on this grid, at high relative humidity, microphysical processes are more likely to be activated than the convection scheme.

\section{Experiment design}

The HWRF model used in this experiment was configured similarly to the 2016 operational system (hereafter referred to as H6CL or Control), with the exception that the operational one-way wave coupling (WAVEWATCH III) was not used. The atmospheric model uses the WRF Nonhydrostatic Mesoscale Model (NMM) dynamic core and was configured with a parent domain and two storm-following, telescopic nested grids. The parent grid $(\mathrm{d} 01)$ had $288 \times 576$ grid points and covered an $80^{\circ} \times 80^{\circ}$ area with $0.135^{\circ}$ (approximately $18 \mathrm{~km}$ ) horizontal grid spacing. The middle grid (d02) covered a $25^{\circ} \times 25^{\circ}$ area with $0.045^{\circ}$ (approximately $6 \mathrm{~km}$ ) grid spacing. The third grid (second nest, d03) had an area of $8.3^{\circ} \times 8.3^{\circ}$ with $0.015^{\circ}$ (about $2 \mathrm{~km}$ ) grid intervals. The two moving nests ( $\mathrm{d} 02$ and $\mathrm{d} 03$ ) were two-way interactive with $50 \%$ feedback and followed the tropical cyclone. Both parent and nests used the WRFNMM rotated latitude-longitude projection and the E-staggered grid. The location of the parent and nests varied from run to run and was dictated by the location of the storm at the initialization time and by the 72-h forecast position, as provided by the National Hurricane Center's TC vitals. Sixty vertical levels (61 sigma entries) were employed, with a model top of $2 \mathrm{hPa}$. Additional intermediate domains were used for the atmospheric model during the vortex relocation, initialization, and postprocessing procedures (Biswas et al. 2018).

The control configuration follows the operational HWRF physics suite, shown in Table 2. The experimental configuration (H6GF) used an alternate CPS. 
TABLE 2. Configuration details for the model experiments.

\begin{tabular}{lll}
\hline \multicolumn{1}{c}{ ATCF ID } & \multicolumn{1}{c}{ H6CL } & \multicolumn{1}{c}{ H6GF } \\
\hline Cumulus & Scale-aware SAS & Grell-Freitas \\
Microphysics & Ferrier-Aligo & Ferrier-Aligo \\
Radiation LW/cloud overlap & RRTMG/maximum random & RRTMG/maximum random \\
Radiation SW/cloud overlap & RRTMG/maximum random & RRTMG/maximum random \\
PBL & GFS-EDMF & GFS-EDMF \\
Surface layer & GFDL & GFDL \\
Model dynamics time step (s) for d01, d02, d03 & $30,10,3.33$ & $30,10,3.33$ \\
Resolution $(\mathrm{km})$ & $18,6,2$ & $18,6,2$ \\
\hline
\end{tabular}

All other components of the operational physics suite were the same for each experiment. Details of each configuration are included in Table 2.

Forecasts for H6CL and H6GF were initialized every $6 \mathrm{~h}$ for Hurricanes Edouard (2014), Gonzalo (2014), and Matthew (2016), and run out to $126 \mathrm{~h}$. The HWRF was initialized with GFS and Global Data Assimilation System (GDAS) data. A regional hybrid ensemble-3DVar data assimilation scheme in Gridpoint Statistical Interpolation (GSI) was utilized to assimilate additional observations (Biswas et al. 2018). A cold start initialization was employed for the first case of each storm, and the HWRF vortex was cycled for all subsequent initializations of a given storm. The cycled run is performed if a previous 6-h forecast is present. The vortex is extracted from the forecast and corrected based on the observed estimate to be used for the current initialization (Biswas et al. 2018). In an effort to increase storm diversity with limited computational resources, 126 -h forecasts were run every $18 \mathrm{~h}$, with 12 -h forecasts for all intermediate initializations for cycling purposes. As an example, 0000 UTC was run for $126 \mathrm{~h}$, 0600 and 1200 UTC were run for $12 \mathrm{~h}$ for cycling purposes, $1800 \mathrm{UTC}$ was run for $126 \mathrm{~h}$. The 18 -h interval was chosen to get a good mixture of different synoptic times at initializations. There were 128 homogeneous cases between the HWRF forecasts with the SAS scheme (H6CL) and those with the GF scheme (H6GF). Since the $126-\mathrm{h}$ forecasts were conducted every $18 \mathrm{~h}$, the number of verified cases at $18 \mathrm{~h}$ was 41 .

\section{Results}

\section{a. Track and intensity statistics}

The forecast storm location and intensity (maximum $10-\mathrm{m}$ wind), as contained in the files produced by the GFDL tropical cyclone tracker, were compared against the best track using the Model Evaluation Tools Tropical Cyclone tool (METTC; Fowler et al. 2017). The METTC tool was run for each case, at 6-h forecast lead times, out to $120 \mathrm{~h}$, to generate a distribution of errors. All metrics are accompanied by $95 \%$ confidence intervals to describe the uncertainty in the result due to sampling limitations. Pairwise differences of track error and absolute intensity error were computed using an R-based statistical language script, where differences between the configurations are considered statistically significant (SS) based on $95 \%$ confidence intervals on the median. All metrics were computed using a homogeneous sample.

Track errors for all three TCs indicate SS differences between the H6GF and H6CL for some of the early lead times (18-h improvement, and 48- and 54-h degradation), whereas mean differences show non-SS smaller track errors for H6GF beyond $84 \mathrm{~h}$ (Fig. 1a). The mean intensity bias is closer to zero in H6GF than in H6CL, but the differences are not SS (Fig. 1c). The operational HWRF is known to underpredict intensity for strong AL storms, which are heavily represented within this sample. The H6GF configuration mitigates this tendency for the storms in this sample. The sudden drop in number of cases from 0 to $18 \mathrm{~h}$ is attributed to the initialization of full 5-day forecasts every $18 \mathrm{~h}$, as described above. An analysis of rapid intensification (RI), which is usually defined as a 30 -kt $\left(1 \mathrm{kt} \approx 0.51 \mathrm{~m} \mathrm{~s}^{-1}\right)$ increase in maximum sustained wind speed in $24 \mathrm{~h}$, shows that the probability of detection (POD) is the same for both configurations. However, if the intensity change threshold is decreased to 10,15 , and $20 \mathrm{kt}(24 \mathrm{~h})^{-1}$, the POD for H6GF is larger, indicating that $\mathrm{H} 6 \mathrm{GF}$ tends to predict moderate intensity changes better than H6CL (Table 3). This is also evident from the alleviation of the negative intensity bias at shorter lead times compared to the control (Fig. 1b). Although the false alarm ratio (FAR) is higher in H6GF (0.77) than in H6CL (0.44), the overall score (i.e., critical success index) for RI prediction is similar in H6GF (0.08) and H6CL (0.10).

\section{b. Case study}

To investigate the difference in TC structure forecasts between the two configurations, a case (1200 UTC 13 October 2014) with a similar track forecast was chosen, so the impact of the location errors was minimized. The case was also representative of the overall intensity 
(a)

\section{Mean Track Error}

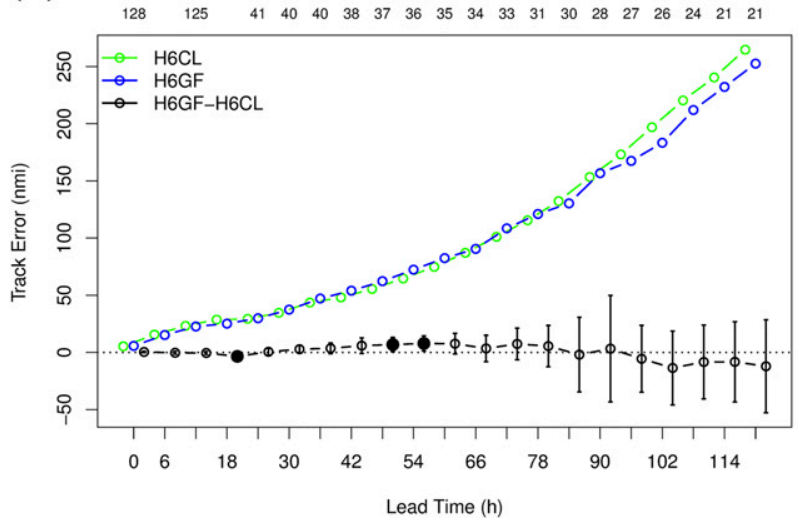

(c)

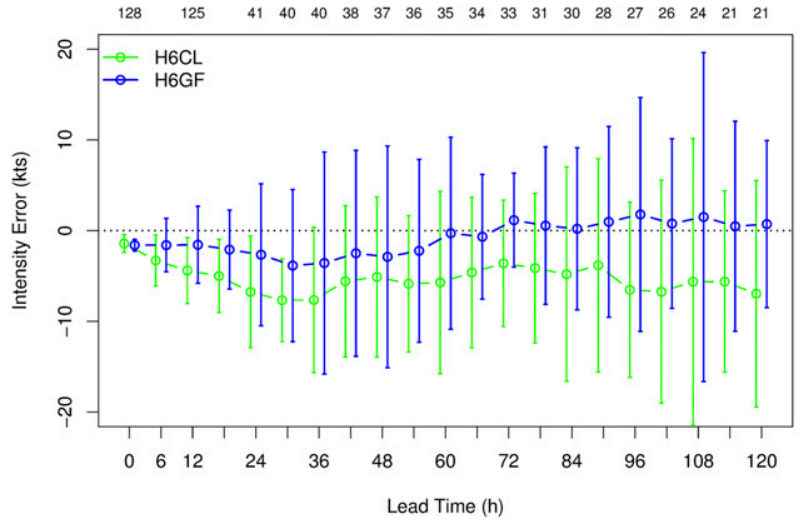

mean errors. Figures $2 \mathrm{a}$ and $2 \mathrm{~b}$ shows the 126 -h track and intensity forecasts of Hurricane Gonzalo initialized at 1200 UTC 13 October 2014, along with the best track. The forecast tracks were very similar to the observed one; however, the intensity forecasts differed dramatically (Fig. 2b). Figure $2 b$ shows that H6CL was unable to intensify the storm after $24 \mathrm{~h}$. Both configurations were able to predict the first episode $(0-24 \mathrm{~h})$ of rapid intensification, but H6CL failed to predict the second episode $(6-30 \mathrm{~h})$, while H6GF was able to predict it with fairly good accuracy. Though there were no other rapid intensification episodes in this forecast, H6GF intensified until $60 \mathrm{~h}$. However, H6CL failed to intensify beyond $24 \mathrm{~h}$.

\section{1) CASE STUdy SHOWING THE DIFFERENCE IN TIME MEAN CROSS SECTIONS OF $V T, V R$, HUMIDITY, CONVERGENCE, AND DIABATIC HEATING}

A comparison of the tangential $V_{t}$ and radial $V_{r}$ velocities between H6CL and H6GF is shown in Fig. 3. These quantities are time averaged over the $6 \mathrm{~h}$ leading up to the intensification at the 24-h lead time. The two forecasts diverge thereafter. The H6GF exhibited

\section{(b) Absolute Intensity Error}

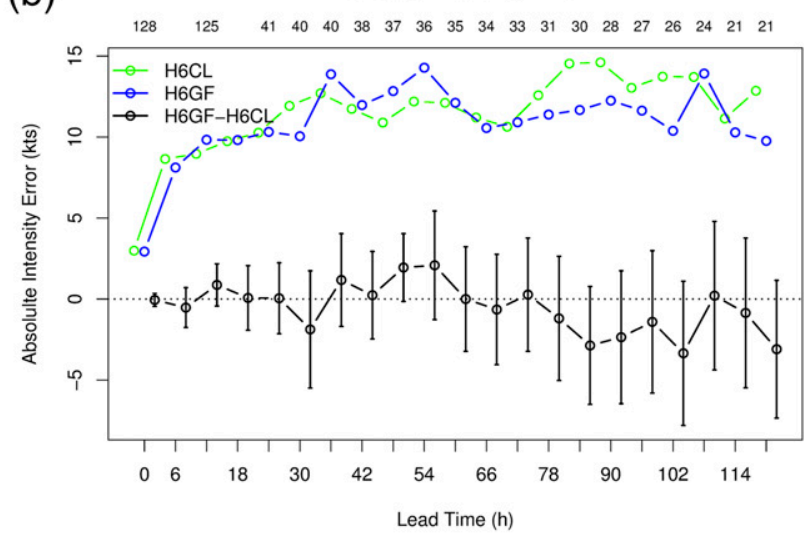

FIG. 1. (a) Mean track errors (n mi), (b) absolute intensity errors (kt), and (c) mean intensity errors (kt) with respect to lead time. H6CL (operational) is in green, H6GF in blue, and pairwise differences (H6GF - H6CL) are shown in black with 95\% confidence intervals. Numbers along the top of the plots indicate the number of cases used at each time.

stronger tangential winds, and the radial gradient of those winds was stronger, indicating a more compact vortex (Lee and Bell 2007). The gradient of tangential winds in the eyewall region shows that the vortex was approximately upright below $8 \mathrm{~km}$ for H6GF (Fig. 3a) and $4 \mathrm{~km}$ for H6CL (Fig. 3b). Above the upright vortex, the eyewall sloped outward. The degree of outward slope of the eyewall is linked to the size of the storm (i.e., the radius of maximum wind, or RMW) according to a previous theoretical study (Stern et al. 2014). In our results, when the RMW is smaller, the slope of the eyewall is also smaller, which is consistent with the theory. Zhang et al. $(2015,2017)$ also found similar relationships between the eyewall slope and the RMW in HWRF forecasts while evaluating the impact of the boundary layer parameterization on intensity and structure forecasts. Another difference in the tangential wind field between $\mathrm{H} 6 \mathrm{GF}$ and $\mathrm{H} 6 \mathrm{CL}$ is the magnitude of the maximum tangential wind speed and the depth of the vortex. The maximum $V_{t}$ is much larger in the H6GF forecast than in the H6CL forecast. The vortex in the H6GF forecast is also much deeper than in the H6CL forecast, with the $25 \mathrm{~m} \mathrm{~s}^{-1}$ contour extending to $8 \mathrm{~km}$ in 
TABLE 3. Probability of detection at various intensity increase thresholds.

\begin{tabular}{ccc}
\hline \hline Intensity increase (kt) threshold in 24h & H6CL & H6GF \\
\hline 30 & 0.11 & 0.11 \\
20 & 0.35 & 0.48 \\
15 & 0.49 & 0.53 \\
10 & 0.57 & 0.59 \\
\hline
\end{tabular}

the H6GF forecast, but only to $4 \mathrm{~km}$ in the $\mathrm{H} 6 \mathrm{CL}$ forecast.

The overall structure for the secondary circulation (i.e., radial wind $V r$ ) is similar in the two forecasts, showing the strong inflow in the boundary layer (Figs. 3c,d). The boundary layer depth in terms of the inflow layer, defined as the height of $10 \%$ peak inflow following Zhang et al. (2011), is similar between the two forecasts in the outer core region $(r>100 \mathrm{~km})$. However, it is evident that the inflow layer is much shallower close to the eyewall region in the H6GF forecast than in the H6CL forecast. The peak inflow in the boundary layer is $2-4 \mathrm{~m} \mathrm{~s}^{-1}$ larger in the H6GF forecast than in the H6CL forecast. Interestingly, the outflow immediately above the inflow layer near the eyewall region is much larger in the H6CL forecast than in the H6GF forecast, which acts to spin down the vortex according to theory (Montgomery et al. 2001). The magnitude of the outflow at upper levels in the outflow layer is also larger in the H6CL forecast than in the H6GF forecast, which may be due to the stronger midlevel inflow in the H6CL forecast.

To verify the TC structure in the two forecasts, we compare the forecasts to the Doppler radar data (Fig. 4). A NOAA P3 flight was conducted on 15 October, centered at 1445 UTC, and wind data were collected using a Doppler radar. The wind speed at $2-\mathrm{km}$ height is shown for H6CL and H6GF (Figs. 4a,b), and contrasted with a radar swath centered at the same time as the model simulation (Fig. 4c). In terms of the maximum wind speed, H6GF performed much better than H6CL compared to the radar observations. Both forecasts captured the asymmetry of the wind field, but the wind field is generally too weak in the H6CL forecast near the eyewall region. In addition, the Doppler radar data show a larger degree of asymmetry of the wind field than both H6GF and H6CL. Both forecasts show larger storm size in terms of the RMW than the observation $(24 \mathrm{~km}$ for H6GF and $26 \mathrm{~km}$ for $\mathrm{H} 6 \mathrm{CL}$ ), with the RMW in the H6GF forecast being closer to the observed value $(20 \mathrm{~km})$.

\section{2) ANALYSIS OF ANGULAR MOMENTUM}

A budget analysis of the absolute angular momentum $M$ was conducted to evaluate why the intensity forecasts
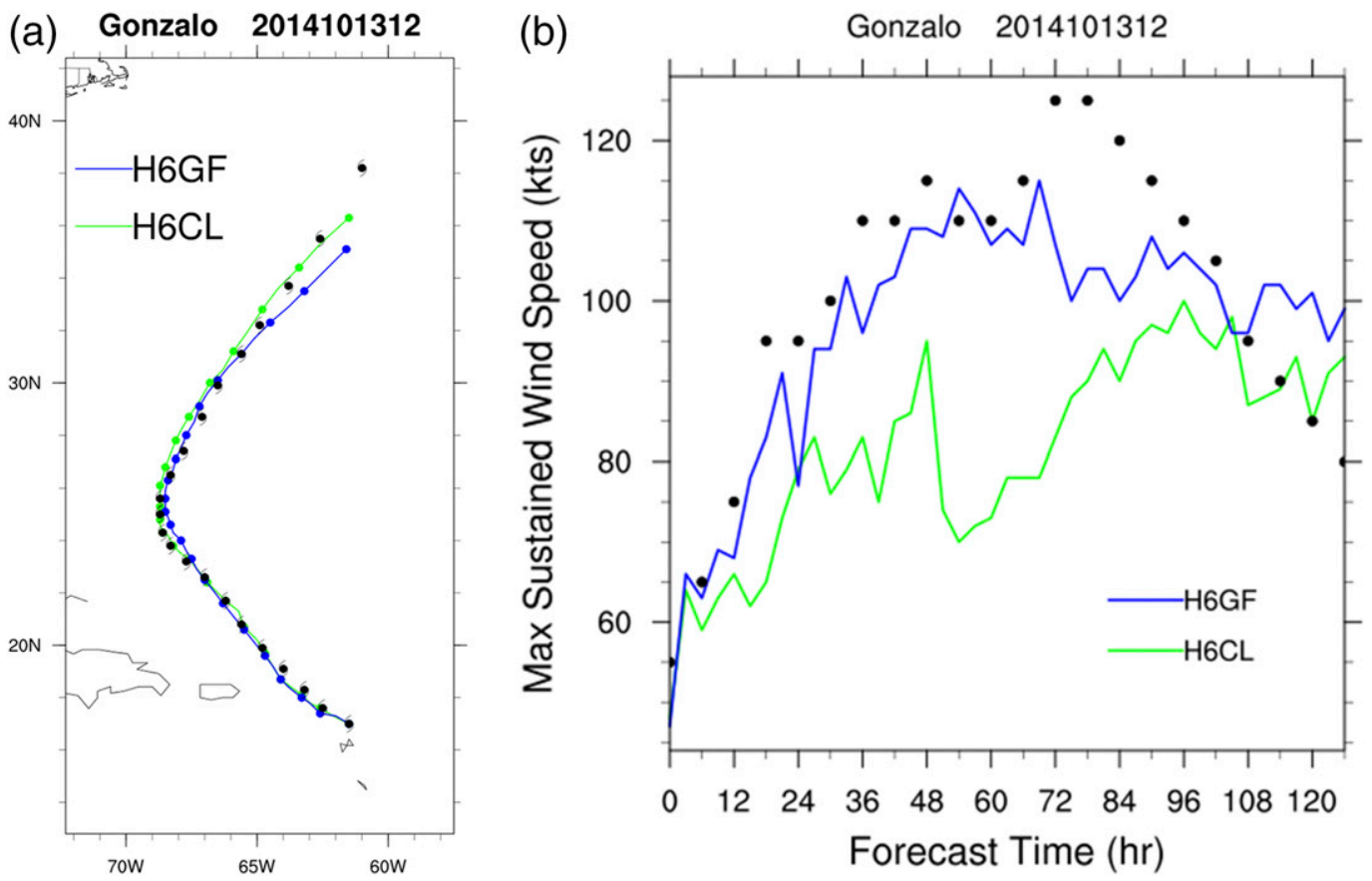

FIG. 2. The 126-h forecast of (a) track and (b) intensity (kt) of Hurricane Gonzalo initialized at 1200 UTC 13 Oct 2014. The black hurricane symbols indicate the best track, green indicates H6CL, and blue indicates H6GF forecasts. 

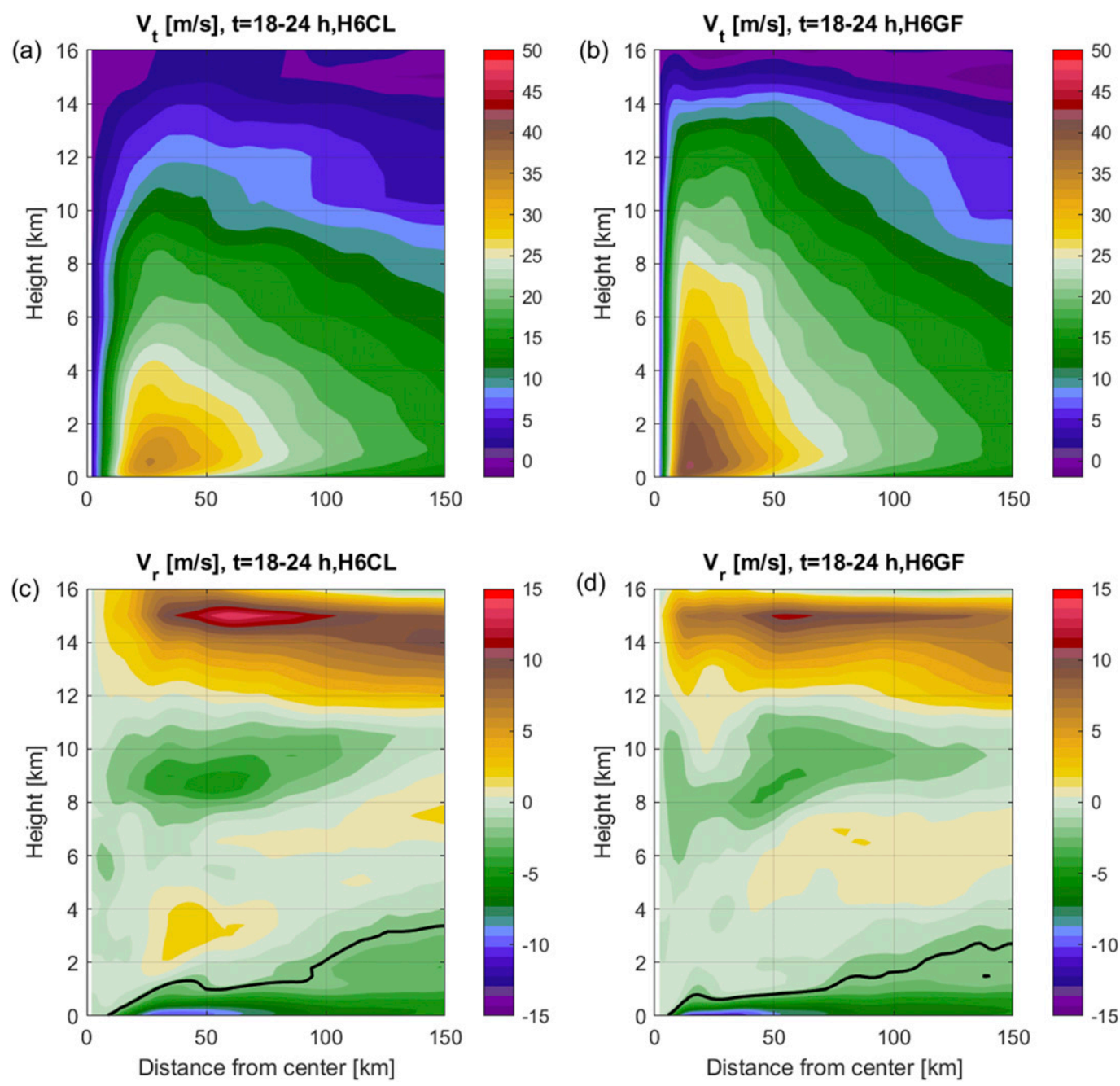

FIG. 3. Azimuthally averaged tangential wind speed for (a) H6CL and (b) H6GF; radial wind velocity for (c) H6CL and (d) H6GF as a function of distance from the center of the storm and height averaged during 18-24 h. The solid lines in (c) and (d) represent the inflow layer depth defined as the height of the $10 \%$ peak inflow.

of Gonzalo in H6GF and H6CL are different. Here $M=$ $r V_{t}+2 f r^{2}$, where $r$ is radial distance and $f$ is the Coriolis frequency. The interpretation of the angular momentum budget parallels that of Zhang and Marks (2015), with small changes. The budget equation for the azimuthally averaged $M$ tendency has the form:

$$
\begin{aligned}
\frac{\partial\langle M\rangle}{\partial t}= & -\left\langle V_{r}\right\rangle \frac{\partial\langle M\rangle}{\partial r}-\langle w\rangle \frac{\partial\langle M\rangle}{\partial z}-\left\langle V_{r}^{\prime} \frac{\partial M^{\prime}}{\partial r}\right\rangle \\
& -\left\langle w^{\prime} \frac{\partial M^{\prime}}{\partial z}\right\rangle+F_{r},
\end{aligned}
$$

where $w$ is the vertical velocity. The bracket denotes an azimuthal average at a given height, and the prime represents a departure from the azimuthal mean (or "eddy" term). Here, the three velocity components are storm relative. Terms on the right-hand side of Eq. (1) are the mean radial advection of $\langle M\rangle$, the mean vertical advection of $\langle M\rangle$, the radial eddy transport of $\langle M\rangle$, the vertical eddy transport of $\langle M\rangle$, and the residual term $F_{r}$, respectively. This equation is the same as in the angular momentum budget of Smith et al. (2009), except that the eddy transport terms are shown here. The residual term $F_{r}$ is calculated by subtracting the advection terms from the tendency term. Both the vertical and horizontal diffusion of angular momentum are included in the residual term, as well as other unresolved processes to close the budget. Detailed output of the diffusion terms from a research version of the HWRF model are presented in Smith et al. (2017), but these were not output in this study to save disk space across multiple retrospective forecasts.

The impact of mean and eddy transport terms on the total $\langle M\rangle$ tendency is first compared between the two 

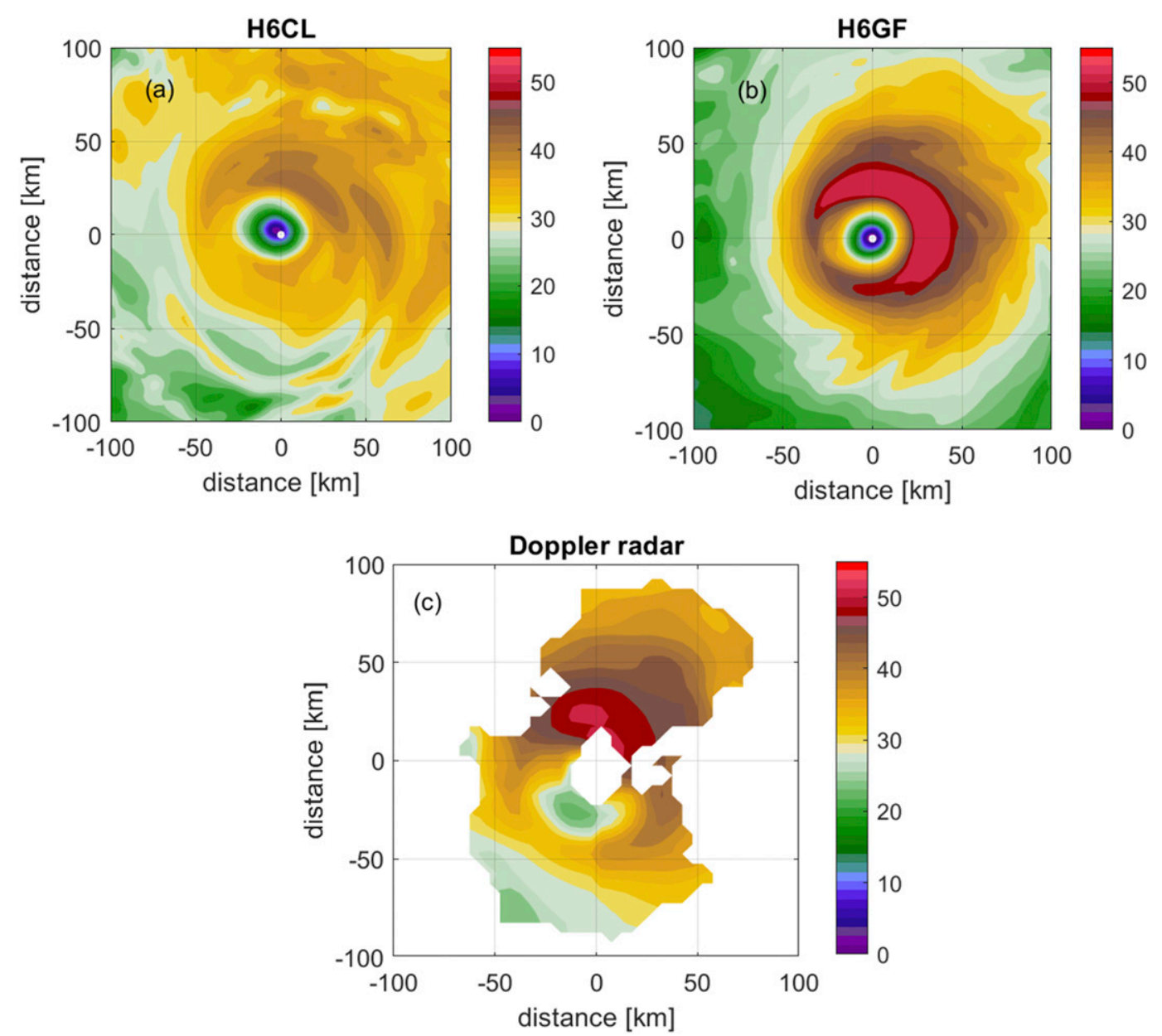

FIG. 4. Wind speed $\left(\mathrm{m} \mathrm{s}^{-1}\right.$ ) at a height of $2 \mathrm{~km}$ above ground level for (a) H6CL, (b) H6GF, and (c) Doppler radar centered at 1445 UTC 15 Sep 2014.

forecasts. Figure 5 shows the tendency of $\langle M\rangle$, the total mean and eddy transport of $\langle M\rangle$, and the $F_{r}$ term from the H6CL and H6GF forecasts. As for the previously shown cross sections, the momentum budget is presented for the 18-24-h forecasts, corresponding to the time when the intensity forecasts start to diverge. The budget terms shown in Fig. 5 are the mean values for this period. It is evident that the tendency term is much larger in the H6GF forecast than in the H6CL forecast (Figs. 5a,b). In both the H6CL and H6GF forecasts, the total mean advection of $\langle M\rangle$ contributes more to the total tendency than the eddy advection of $\langle M\rangle$. However, the mean advection term is much larger in the H6GF forecast than in the H6CL forecast (Figs. 5c,d). This difference is particularly large in the boundary layer between the two forecasts, and is mainly attributed to the difference in the strength of the inflow. As shown earlier in Fig. 3, the magnitude of the low-level inflow is much larger in the H6GF forecast than in the H6CL forecast.
The eddy contribution to the total $\langle M\rangle$ tendency in the two forecasts shows similar results in that this term generally reduces the total tendency, especially inside the RMW at low levels $(<4 \mathrm{~km})$. However, the eddy contribution to the total tendency is less negative in H6GF than in H6CL within the eyewall at low levels. At midlevels $(6-8 \mathrm{~km})$, the eddy term shows positive values in the eyewall region in both forecasts. The overall difference in the eddy term between the two forecasts is small. But the negative eddy advection is overcome by the larger positive mean advection in the H6GF forecast, which results in a positive total tendency, while in H6CL the magnitude of the negative eddy advection is actually larger than that of the positive mean advection, which results in a negative total tendency in the eyewall. Thus, the difference in the total spinup rate of the vortex in the two forecasts is mainly due to the difference in the mean advection term.

Of note, in H6GF, the spin up of the vortex in the boundary layer is largely due to the radial advection 

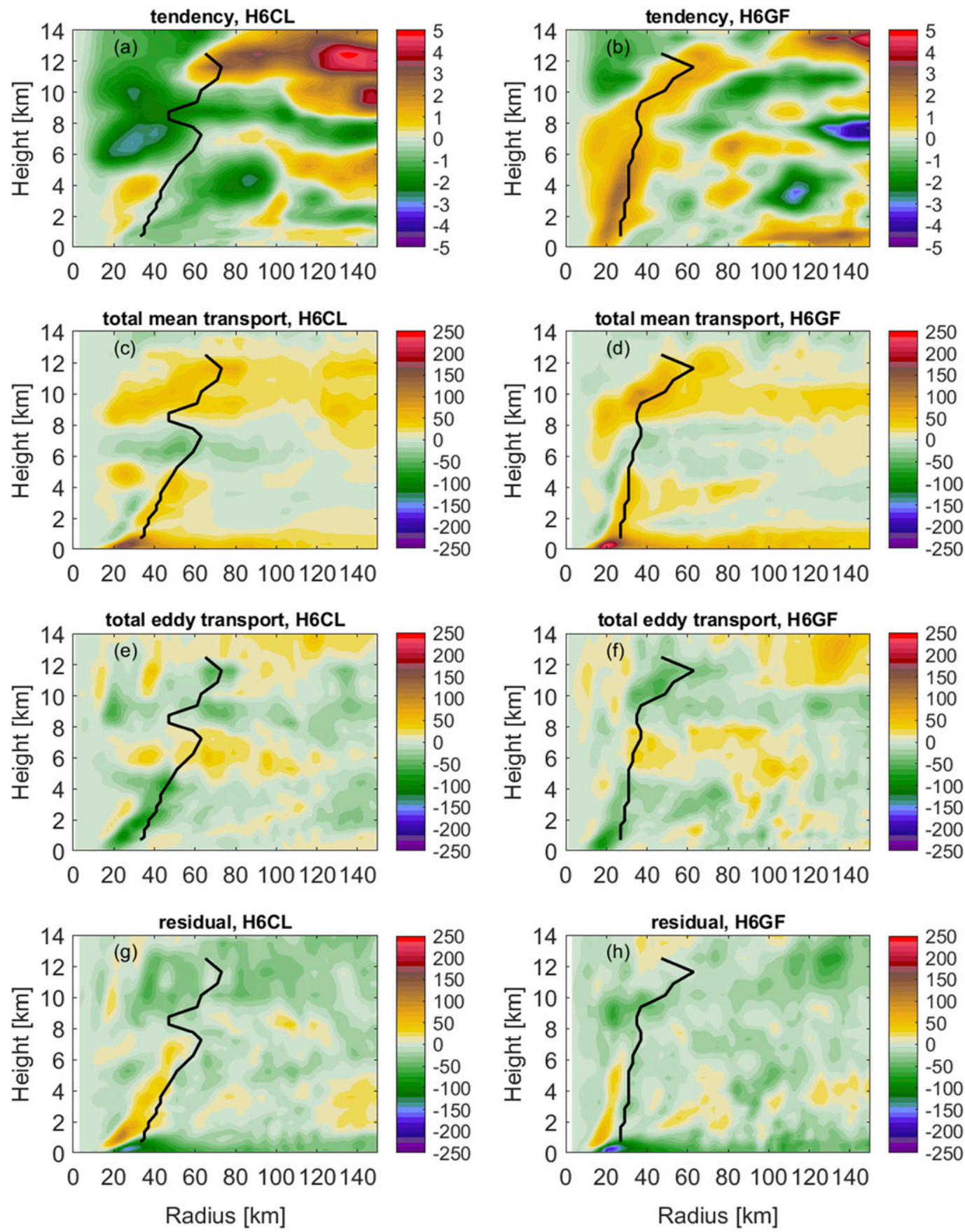

FIG. 5. Radius-height plots of the terms in the azimuthally averaged absolute angular momentum $\langle M\rangle$ budget for simulations with (left) H6CL and (right) H6GF schemes during the 18-24-h period. These budget terms $\left(\mathrm{m}^{2} \mathrm{~s}^{-2}\right)$ include (a),(b) the local rate of change of $\langle M\rangle$; (c),(d) the total mean advection; (e),(f) the sum of the eddy transport of $\left\langle M^{\prime}\right\rangle$; and $(\mathrm{g}),(\mathrm{h})$ the residual term $F_{r}$. The black line represents the radius of maximum azimuthally averaged tangential wind speed. Note the different color scales in (a) and (b) vs (c)-(h).

of $\langle M\rangle$. Above the boundary layer, the mean advection is mainly due to the vertical advection of $\langle M\rangle$ (not shown). The radial advection of $\langle M\rangle$ exceeds the boundary layer frictional effect in the residual term in the H6GF forecast when the storm intensifies, which agrees with the spinup theory of Smith et al. (2009). It is the stronger boundary layer inflow in the H6GF forecast that makes the radial advection of large $\langle M\rangle$ much larger in the H6GF forecast than in the H6CL forecast, so that Hurricane Gonzalo spins 

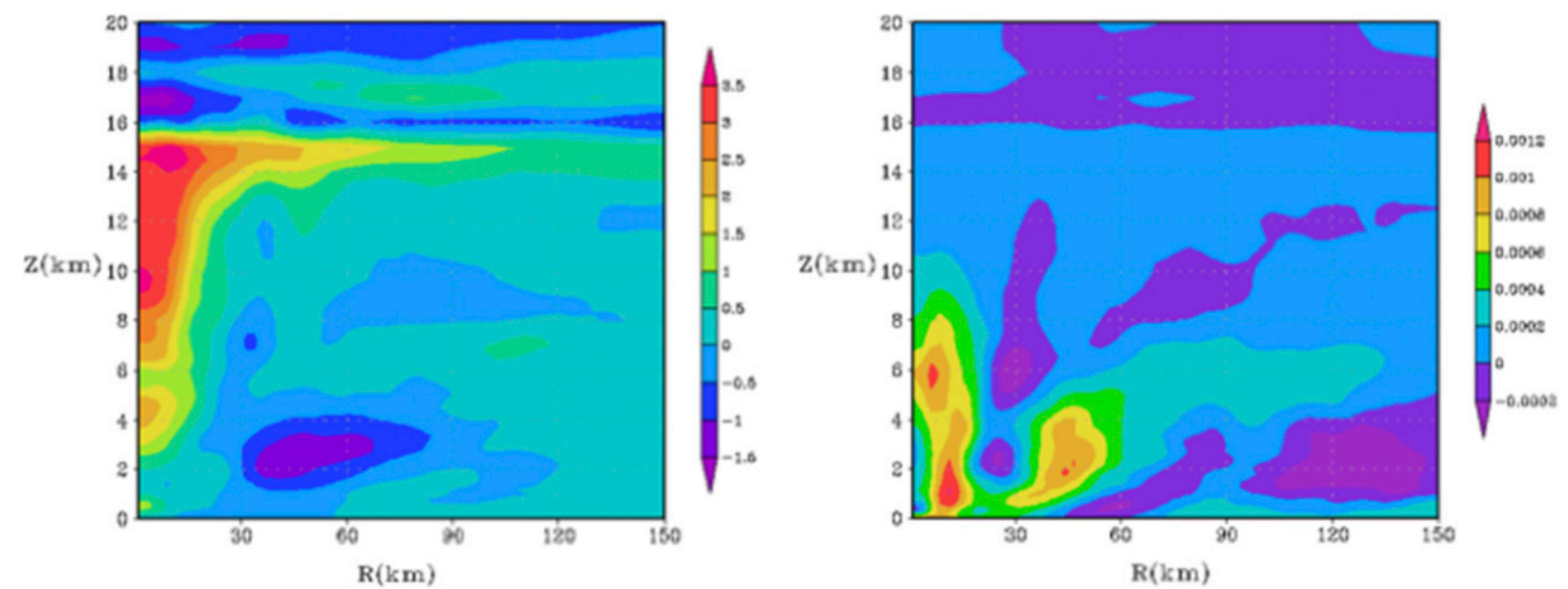

FIG. 6. Difference between H6GF and H6CL azimuthally averaged (a) temperature $\left({ }^{\circ} \mathrm{C}\right)$ and (b) specific humidity $\left(\mathrm{kg} \mathrm{kg}^{-1}\right)$ between 18 and $24 \mathrm{~h}$.

up much faster in the H6GF forecast than in the H6CL forecast. This angular momentum budget explains why H6GF performed better than H6CL in terms of the spinup process to reach the observed storm intensity.

\section{3) THERMODYNAMIC STRUCTURE AND BUDGET ANALYSIS}

To better understand the cause of the kinematic differences shown in the previous section, we next examine the differences in the thermodynamic structure between the H6GF and H6CL forecasts. It is evident from Fig. 6a that the core of Hurricane Gonzalo is much warmer in the H6GF forecast than in the H6CL forecast, indicating a larger warm-core anomaly, which is consistent with the greater storm intensity in the H6GF forecast. The air in the region outside the eyewall immediately above the boundary layer is cooler in the H6GF forecast than in the H6CL forecast, which may be due to the stronger outflow seen in the H6CL forecast (cf. Fig. 3d) and the difference in the boundary layer height between the two forecasts. The inner-core region has higher humidity in the H6GF forecast than in the H6CL forecast, especially in the boundary layer. This result is consistent with that of Zhang et al. (2017) and Kieu et al. (2014), who also found that stronger and more rapidly intensifying TCs have moister boundary layers.

A closer look at the heating due to moist processes in the two model runs during the time period prior to that used in the angular momentum analysis is provided in Figs. 7 and 8. In these figures, the temperature tendencies from the cumulus parameterization and from the microphysical parameterization were averaged in time over $6-18 \mathrm{~h}$, and also averaged in space. It is evident from Figs. 7a-c that both CPS exhibit scale awareness, as the heating due to convection decreases in magnitude from coarser to finer resolutions, since at smaller grid spacings more of the convective processes are resolved. The shape of the convective heating profiles differs, with the SAS scheme producing more diabatic heating at lower levels, while the GF scheme produces a heating maximum near $550 \mathrm{hPa}$. The height of the heating maximum in the GF scheme is strongly influenced by the parameters that describe the PDF of the normalized vertical mass flux.

Figures $7 \mathrm{~d}-\mathrm{f}$ shows the microphysical temperature tendencies. While the profiles are generally similar in shape and magnitude for domains 2 and 3, the magnitudes are quite different on d01. At the highest resolution (d03), the GF scheme has very slightly more low-level heating due to microphysics within the $1^{\circ}$ latitude-longitude averaging area. On the coarser domains, however, the H6GF run exhibits greater microphysical cooling. Diabatic cooling in the microphysics results primarily from evaporation of cloud droplets and from the melting of frozen hydrometeors. Detrainment of cloud water from the convective parameterization can be a significant source of these particles.

From Fig. 6, the temperature and moisture difference between the two runs is located primarily near the storm center. By comparing the average diabatic heating in the inner core region (Fig. 8), it can be seen that the H6GF run has much more heating due to microphysics compared to the H6CL run within this inner core. This indicates greater levels of saturation, as the microphysics scheme is activated only when conditions are saturated. Because the microphysics is linked to gridscale saturation and resolved vertical 

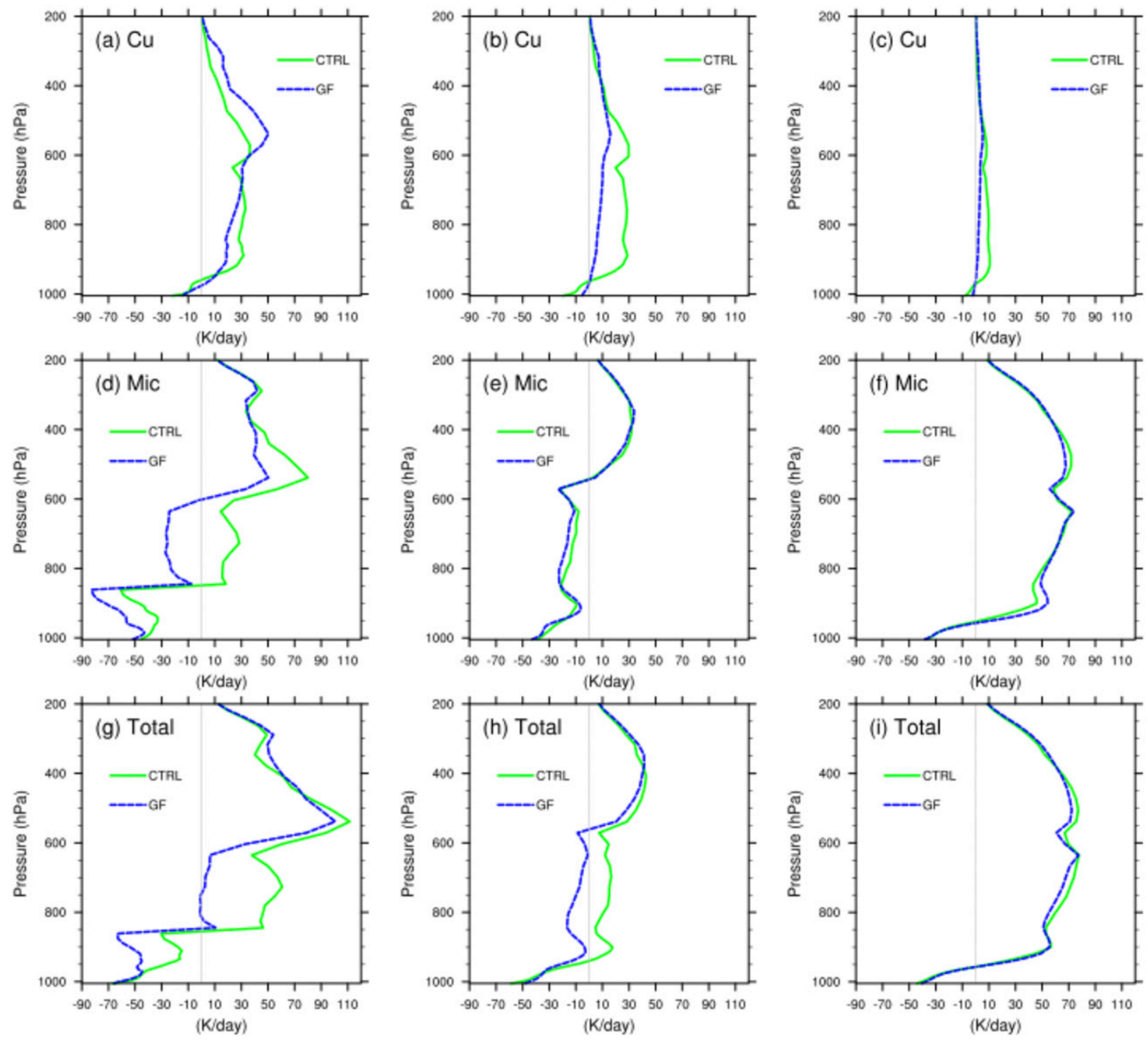

FIG. 7. Temperature tendency $\left(\mathrm{K} \mathrm{day}^{-1}\right)$ averaged over a $1^{\circ}$ radius of latitude-longitude from the center of the storm, and averaged in time for 6-18 h for the H6CL run (green) and the H6GF run (blue). (left) Domain 1, (center) domain 2, and (right) domain 3. (a)-(c) The contribution from the cumulus parameterization, (d)-(f) the contribution from the microphysics, and (g)-(i) the sum of the cumulus and microphysics tendencies.

motion, stronger heating by the microphysical parameterization supports stronger intensification. The larger microphysics contribution also leads to larger precipitation amounts in the H6GF forecast (not shown). In both simulations, the microphysics component of the diabatic heating (Fig. 8b) is far greater than that due to the convective parameterization (Fig. 8a). At 2-km resolution, d03 is considered to be of a convection-permitting scale, at which the resolved physical processes should provide a better representation of the storm evolution.

Although the SAS CPS in H6CL produced more lowlevel heating, the GF CPS led to greater intensification.
This can be attributed to the balance of the microphysics and the cumulus parameterizations. The gridscale processes generally have a stronger feedback to the dynamics compared to the subgrid-scale processes, through the diabatic heating/cooling and vertical motion fields. In sensitivity tests with Hurricane Maria simulations, adjusting the PDF of the normalized mass flux so that the maximum occurred at a lower vertical level led to a weaker storm (not shown).

In addition, heating from the convection scheme is associated with drying, as water vapor is converted to cloud water and precipitation. The low-level heating 

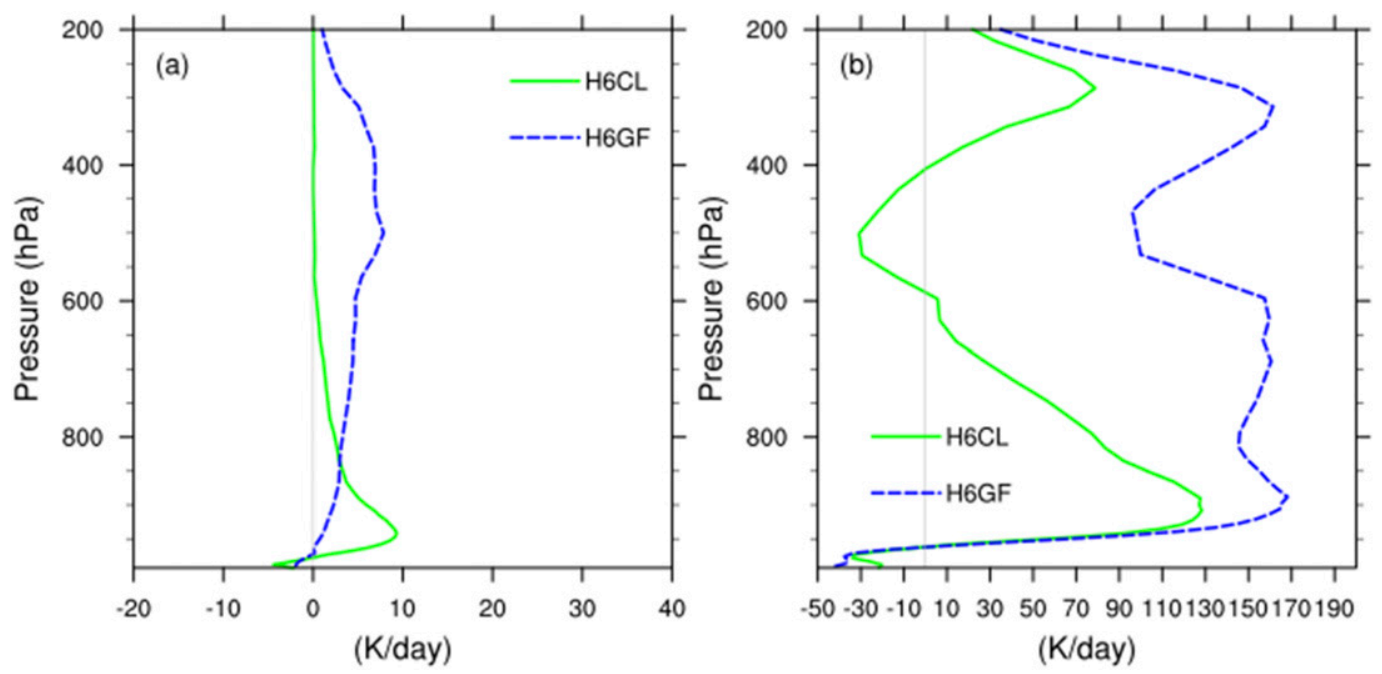

FIG. 8. Temperature tendencies $\left(\mathrm{K} \mathrm{day}^{-1}\right)$ averaged over a $0.25^{\circ}$ latitude-longitude radius from the center of the storm and averaged in time for $6-18 \mathrm{~h}$ for domain 3 of the H6CL and H6GF model runs. (a) The contribution from the cumulus parameterization; (b) the contribution from the microphysics.

maximum from the CPS in the H6CL run during 6-18h may have contributed to the lower specific humidity shown in Fig. 6 b.

To get a more thorough understanding of how the two convective schemes differ, a thermodynamic budget analysis is also conducted to identify the differences in the warm core between H6GF and H6CL. We computed the azimuthal mean of temperature tendency from total advection $\theta_{\text {adv }}$ (horizontal and vertical) using the following equation:

$$
\theta_{\mathrm{adv}}=-u \frac{d \theta}{d r}-w \frac{d \theta}{d z}-u^{\prime} \frac{d \theta^{\prime}}{d r}-w^{\prime} \frac{d \theta^{\prime}}{d z}
$$

The azimuthal average of temperature tendency $\theta_{\text {ten }}$ from total advection $\theta_{\text {adv }}$, diabatic heating (DHEAT), PBL (PBL) and horizontal diffusion (HDIFF) is also calculated using the following equation:

$$
\theta_{\text {ten }}=\theta_{\text {adv }}+\text { DHEAT }+ \text { PBL }+ \text { HDIFF }
$$

The diabatic heating and PBL tendency was available through WRF-NMM output. Note that the PBL tendency is mainly contributed by the vertical diffusion process. The horizontal diffusion term is calculated as following:

$$
\mathrm{HDIFF}=\frac{1}{r} \frac{\partial}{\partial r}\left(r K_{H} \frac{\partial \theta}{\partial r}\right),
$$

where $K_{H}$ is horizontal eddy diffusivity (Zhang and Marks 2015), $r$ is the radial distance, and $\theta$ is temperature. Figure 9 shows the azimuthal averages of all the four terms described above. This temperature budget analysis follows Stern and Zhang (2013). The left panel shows the tendencies from H6CL, and the right panel shows the tendencies from H6GF. Advection is the dominant term. The advection in H6GF is larger than in H6CL near the storm center, which helps develop the stronger warm core. This is also true for the diabatic heating. The largest diabatic heating is located at higher levels between 7 and $11 \mathrm{~km}$ in H6GF, which is associated with deeper convective activities compared to H6CL. The diabatic heating inside the RMW at low levels is much larger in the H6GF forecast than in $\mathrm{H6CL}$, which has been shown to favor intensification (Vigh and Schubert 2009; Smith and Montgomery 2016). The diabatic heating is linked to secondary circulation due to balanced dynamics. Also, according to both the energy efficiency argument and angular momentum transport processes (Nolan et al. 2007; Smith and Montgomery 2016), enhanced diabatic heating inside the RMW could help spin up the vortex above the boundary layer and enhance the warm-core development, in turn the intensity is stronger with larger and deeper diabatic heating. This is consistent with the deep vortex seen in the H6GF forecast (cf. Fig. 3a). The PBL and horizontal diffusion contributions to the total temperature tendency in H6GF are also slightly larger than in H6CL, although of the contributions from the other terms (i.e., advection and diabatic heating) are dominant.

\section{Summary and conclusions}

In this study, we evaluated the impact of two different CPSs on forecasts of TC intensity and structure using HWRF retrospective forecasts. The GF cumulus 

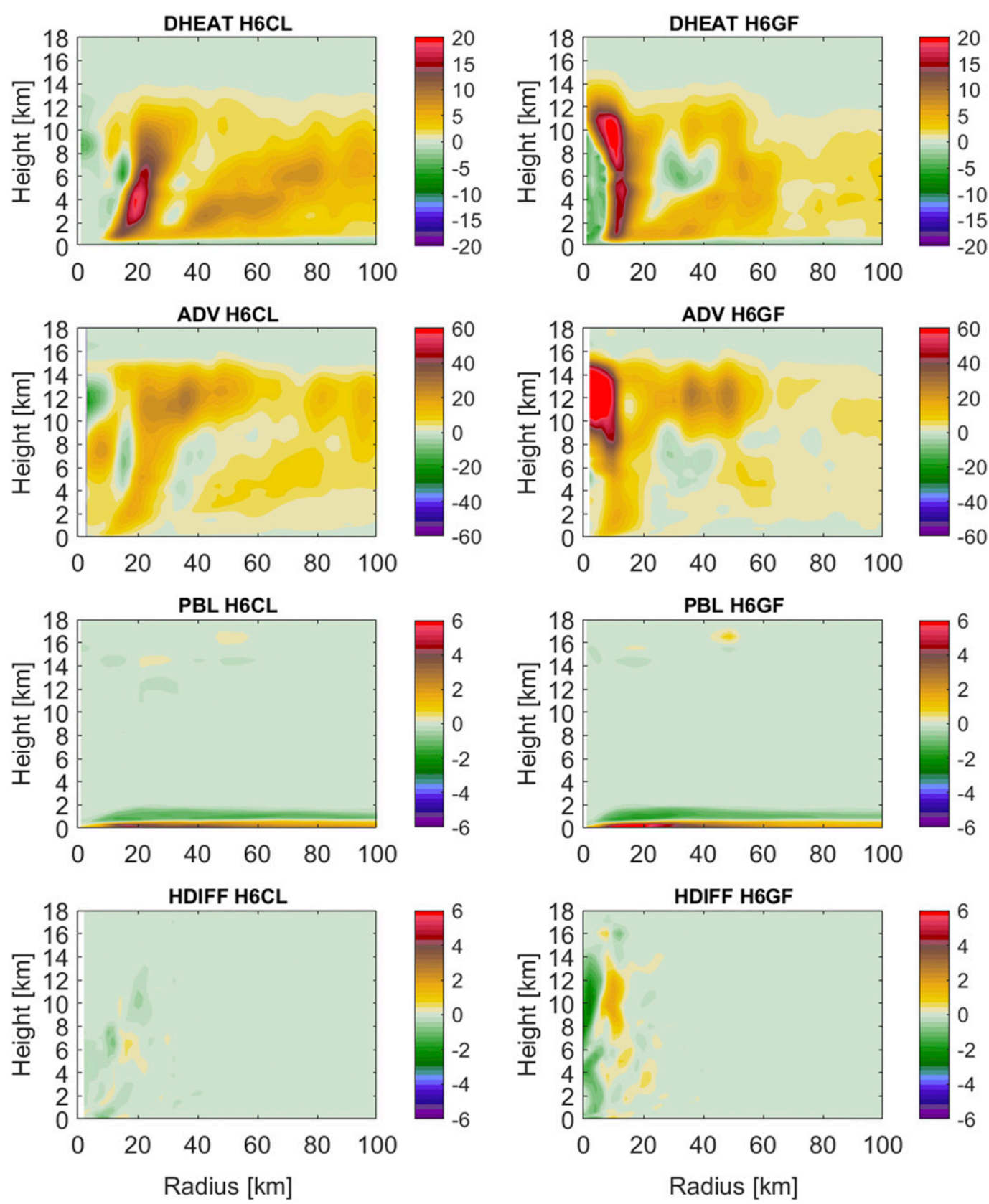

FIG. 9. Radius-height plots of the terms in the azimuthally averaged temperature tendency $\left(\mathrm{Kh}^{-1}\right)$ due to diabatic heating (DHEAT), advection (ADV), PBL parameterization, and horizontal diffusion (HDIFF) for simulations with (left) H6CL and (right) H6GF schemes during the 18-24-h period.

scheme was implemented in HWRF and tested against the performance of the existing scale-aware SAS scheme in the operational HWRF. Two sets of HWRF forecasts were run in the same configuration except for the cumulus scheme. Our results show that the GF (H6GF) scheme performed better than the scale-aware SAS (H6CL) scheme in terms of track and intensity forecasts for a majority of forecast lead times for this set of
TCs. The most significant improvement seen in the forecasts with the GF scheme is the reduction of the intensity bias compared to the forecasts with the SAS scheme. Verification of forecast skill of rapidly intensifying storms shows the POD is larger in the forecasts with the GF scheme than with the SAS scheme.

To understand why the GF scheme produced better intensity forecasts than the SAS scheme, a case study 
was used to evaluate the detailed TC structure and dynamics. Comparison of forecasted wind structure to Doppler radar observations shows that the H6GF forecast captured the larger maximum wind speed seen by the radar. The storm size is also forecasted better in the H6GF forecast than in the H6CL forecast.

It was found that the inflow strength is much larger in the boundary layer in the H6GF forecast than in the H6CL forecast. This enhanced inflow increases angular momentum convergence in the H6GF forecast, which results in a faster spinup rate. This result is consistent with the TC spinup theory (Smith et al. 2009). An angular momentum budget analysis shows that the difference in the contribution of the eddy transport to the total angular momentum tendency is small between the two forecasts. The main difference is in the mean transport term, especially in the boundary layer. The vortex in the H6GF forecast is deeper than that in the H6CL forecast. The faster spin up is due to the increased diabatic heating for H6GF and being located inside the RMW (Smith and Montgomery 2016).

The temperature tendency was also compared between the two forecasts before the intensity divergence point. The larger cumulus and microphysics contributions to the temperature tendency in the H6GF forecast relative to the H6CL forecast help explain why the inner core region is moister and warmer in the H6GF forecast. The larger warm-core anomaly and larger moist entropy in the boundary layer support the stronger storm predicted by HWRF with the GF scheme, which is more consistent with observations.

A temperature budget analysis revealed that the higher potential temperature near the storm center in $\mathrm{H} 6 \mathrm{GF}$ can be attributed to much higher temperature advection and diabatic heating, which leads to higher intensification compared to H6CL. The PBL temperature tendency and diffusion was also slightly higher for H6GF; however, the magnitudes were much lower than those of the advection and diabatic heating terms in the budget.

Above all, the case study presented here helps explain the dynamic and thermodynamic reasons for the superior performance of the GF scheme as compared to the scale-aware SAS scheme in HWRF. The intensity and track error verification suggest that the GF scheme has potential for being used in the operational HWRF model or other TC forecast models. But a larger sample that includes weaker storms is needed to recommend GF for operational implementation.

Acknowledgments. This material is based upon work supported by the National Center for Atmospheric Research (NCAR), which is a major facility sponsored by the National Science Foundation (NSF) under
Cooperative Agreement 1852977. The work was funded by National Oceanic and Atmospheric Administration (NOAA) and Hurricane Forecast Improvement Project (HFIP). Jun Zhang was funded by NSF Grants AGS1822128 and ASG1654831, and the Developmental Testbed Center (DTC) visitor program. Evan Kalina and Ligia Bernardet were supported by funding from NOAA Award NA17OAR4320101. James Frimel was supported by funding from NOAA Award NA19OAR4320073. The DTC is funded by the NOAA, the U.S. Air Force (USAF), the NCAR, and the NSF. The Model Evaluation Tools (MET) was developed at the NCAR through grants from the NSF, NOAA, the USAF, and the U.S. Department of Energy (DOE). The computations were carried out on NOAA's Jet supercomputer. The authors thank the three anonymous reviewers for their comments, improving the paper.

Data availability statement. The data used in the study are available from the corresponding author upon reasonable request.

\section{REFERENCES}

Arakawa, A., and W. H. Schubert, 1974: Interaction of a cumulus cloud ensemble with the large-scale environment, Part I. J. Atmos. Sci., 31, 674-701, https://doi.org/10.1175/15200469(1974)031<0674:IOACCE > 2.0.CO;2.

__ and C. M. Wu, 2013: A unified representation of deep moist convection in numerical modeling of the atmosphere. Part I. J. Atmos. Sci., 70, 1977-1992, https://doi.org/10.1175/ JAS-D-12-0330.1.

- J. H. Jung, and C. M. Wu, 2011: Toward unification of the multiscale modeling of the atmosphere. Atmos. Chem. Phys., 11, 3731-3742, https://doi.org/10.5194/acp-11-3731-2011.

Benjamin, S. G., and Coauthors, 2004: An hourly assimilationforecast cycle: The RUC. Mon. Wea. Rev., 132, 495-518, https://doi.org/10.1175/1520-0493(2004)132<0495:AHACTR > 2.0.CO;2.

— , and Coauthors, 2016: A North American hourly assimilation and model forecast cycle: The Rapid Refresh. Mon. Wea. Rev., 144, 1669-1694, https://doi.org/10.1175/MWR-D-15-0242.1.

Biswas, M. K., L. Bernardet, and J. Dudhia, 2014: Sensitivity of hurricane forecasts to cumulus parameterizations in the HWRF model. Geophys. Res. Lett., 41, 9113-9119, https:// doi.org/10.1002/2014GL062071.

_ Forecasting (HWRF) model: 2017 scientific documentation. NCAR Tech. Note NCAR/TN-544-STR, 111 pp., https:// doi.org/10.5065/D6MK6BPR.

Bu, Y. P., R. G. Fovell, and K. L. Corbosiero, 2017: The influences of boundary layer mixing and cloud-radiative forcing on tropical cyclone size. J. Atmos. Sci., 74, 1273-1292, https:// doi.org/10.1175/JAS-D-16-0231.1.

Cangialosi, J. P., 2018: National Hurricane Center forecast verification report: 2017 hurricane season. NOAA/NHC, 73 pp., http:// www.nhc.noaa.gov/verification/pdfs/Verification_2017.pdf.

, and J. L. Franklin, 2017: National Hurricane Center forecast verification report: 2016 hurricane season. NOAA/NHC, 72 pp., http://www.nhc.noaa.gov/verification/pdfs/Verification_2016.pdf. 
Fowler, L. D., W. C. Skamarock, G. A. Grell, S. R. Freitas, and M. G. Duda, 2016: Analyzing the Grell-Freitas convection scheme from hydrostatic to nonhydrostatic scales within a global model. Mon. Wea. Rev., 144, 2285-2306, https://doi.org/ 10.1175/MWR-D-15-0311.1.

Fowler, T., J. Halley Gotway, K. Newman, T. Jensen, B. Brown, and R. Bullock, 2017: The Model Evaluation Tools version 7.0 (METv7.0) user's guide. Developmental Testbed Center, 407 pp., http://www.dtcenter.org/met/users/docs/users_guide/ MET_Users_Guide_v7.0.pdf.

Freitas, S. R., G. A. Grell, A. Molod, M. A. Thompson, W. M. Putman, C. M. Santos e Silva, and E. P. Souza, 2018: Assessing the Grell-Freitas convection parameterization in the NASA GEOS modeling system. J. Adv. Model. Earth Syst., 10, 1266-1289, https://doi.org/10.1029/2017MS001251.

Gall, R., J. Franklin, F. Marks, E. N. Rappaport, and F. Toepfer, 2013: The Hurricane Forecast Improvement Project. Bull. Amer. Meteor. Soc., 94, 329-343, https://doi.org/10.1175/ BAMS-D-12-00071.1.

Grell, G. A., 1993: Prognostic evaluation of assumptions used by cumulus parameterizations. Mon. Wea. Rev., 121, 764-787, https:// doi.org/10.1175/1520-0493(1993)121<0764:PEOAUB > 2.0.CO;2. , and D. Dévényi, 2002: A generalized approach to parameterizing convection combining ensemble and data assimilation techniques. Geophys. Res. Lett., 29, 1693, https://doi.org/ 10.1029/2002GL015311.

— convective parameterization for weather and air quality modeling. Atmos. Chem. Phys., 14, 5233-5250, https://doi.org/ 10.5194/acp-14-5233-2014.

_ J. Dudhia, and D. Stauffer, 1994: A description of the fifthgeneration Penn State/NCAR Mesoscale Model (MM5). NCAR Tech. Note NCAR/TN-398+STR, NCAR, 128 pp., https://doi.org/10.5065/D60Z716B.

Han, J., and H. Pan, 2011: Revision of convection and vertical diffusion schemes in the NCEP global forecast system. Wea. Forecasting, 26, 520-533, https://doi.org/10.1175/WAF-D-10-05038.1.

Kieu, C., V. Tallapragada, and W. Hogsett, 2014: Vertical structure of tropical cyclones at onset of the rapid intensification in HWRF model. Geophys. Res. Lett., 41, 3298-3306, https:// doi.org/10.1002/2014GL059584.

Lee, W.-C., and M. M. Bell, 2007: Rapid intensification, eyewall contraction, and breakdown of Hurricane Charley (2004) near landfall. Geophys. Res. Lett., 34, L02802, https://doi.org/ 10.1029/2006GL027889.

Montgomery, M. T., H. D. Snell, and Z. Yang, 2001: Axisymmetric spindown dynamics of hurricane-like vortices. J. Atmos. Sci., 58, 421-435, https://doi.org/10.1175/1520-0469(2001)058<0421: ASDOHL $>2.0 . \mathrm{CO} ; 2$.

Nasrollahi, N., A. AghaKouchak, J. Li, X. Gao, K. Hsu, and S. Sorooshian, 2012: Assessing the impacts of different WRF precipitation physics in hurricane simulations. Wea. Forecasting, 27, 1003-1016, https://doi.org/10.1175/WAFD-10-05000.1.

Nolan, D. S., Y. Moon, and D. P. Stern, 2007: Tropical cyclone intensification from asymmetric convection: Energetics and efficiency. J. Atmos. Sci., 64, 3377-3405, https://doi.org/10.1175/ JAS3988.1.

Pan, H.-L., and W.-S. Wu, 1995: Implementing a mass flux convective parameterization package for the NMC medium-range forecast model. NMC Office Note 409, $40 \mathrm{pp}$.

Pattanayak, S., U. C. Mohanty, and K. K. Osuri, 2012: Impact of parameterization of physical processes on simulation of track and intensity of tropical cyclone Nargis (2008) with WRFNMM model. Sci. World J., 2012, 1-18, https://doi.org/10.1100/ 2012/671437.

Shepherd, T. J., and K. J. Walsh, 2017: Sensitivity of hurricane track to cumulus parameterization schemes in the WRF model for three intense tropical cyclones: Impact of convective asymmetry. Meteor. Atmos. Phys., 129, 345-374, https://doi.org/ 10.1007/s00703-016-0472-y.

Simpson, J., 1971: On cumulus entrainment and one-dimensional models. J. Atmos. Sci., 28, 449-455, https://doi.org/10.1175/ 1520-0469(1971)028<0449:OCEAOD > 2.0.CO;2.

, R. H. Simpson, D. A. Andrews, and M. A. Eaton, 1965: Experimental cumulus dynamics. Rev. Geophys., 3, 387-431, https://doi.org/10.1029/RG003i003p00387.

Smith, R. K., 2000: The role of cumulus convection in hurricanes and its representation in hurricane models. Rev. Geophys., $\mathbf{3 8}$, 465-489, https://doi.org/10.1029/1999RG000080.

_ , and M. T. Montgomery, 2016: The efficiency of diabatic heating and tropical cyclone intensification. Quart. J. Roy. Meteor. Soc., 142, 2081-2086, https://doi.org/10.1002/qj.2804.

,-- , and N. V. Sang, 2009: Tropical cyclone spin up revisited. Quart. J. Roy. Meteor. Soc., 135, 1321-1335, https://doi.org/ 10.1002/qj.428.

__ J. A. Zhang, and M. T. Montgomery, 2017: The dynamics of intensification in a Hurricane Weather Research and Forecasting simulation of hurricane Earl (2010). Quart. J. Roy. Meteor. Soc., 143, 293-308, https://doi.org/10.1002/ qj.2922.

Stern, D. P., and F. Zhang, 2013: How does the eye warm? Part I: A potential temperature budget analysis of an idealized tropical cyclone. J. Atmos. Sci., 70, 73-90, https://doi.org/ 10.1175/JAS-D-11-0329.1.

— J. J. R. Brisbois, and D. S. Nolan, 2014: An expanded dataset of hurricane eyewall sizes and slopes. J. Atmos. Sci., 71, 2747-2762, https://doi.org/10.1175/JAS-D-13-0302.1.

Sun, Y., Z. Zhong, W. Lu, and Y. Hu, 2014: Why are tropical cyclone tracks over the western North Pacific sensitive to the cumulus parameterization scheme in regional climate modeling? A case study for Megi (2010). Mon. Wea. Rev., 142, 1240-1249, https://doi.org/10.1175/MWRD-13-00232.1.

Tiedtke, M., 1989: A comprehensive mass flux scheme for cumulus parameterization in large-scale models. Mon. Wea. Rev., 117, 1779-1800, https://doi.org/10.1175/1520-0493(1989)117<1779: ACMFSF $>2.0 . C O ; 2$.

Vigh, J. L., and W. H. Schubert, 2009: Rapid development of the tropical cyclone warm core. J. Atmos. Sci., 66, 3335-3350, https://doi.org/10.1175/2009JAS3092.1.

Wang, W., J. A. Sippel, S. Abarca, L. Zhu, B. Liu, Z. Zhang, A. Mehra, and V. Tallapragada, 2018: Improving NCEP HWRF simulations of surface wind and inflow angle in the eyewall area. Wea. Forecasting, 33, 887-898, https://doi.org/ 10.1175/WAF-D-17-0115.1.

Zhang, G. J., and X. Wu, 2003: Convective momentum transport and perturbation pressure field from a cloud-resolving model simulation. J. Atmos. Sci., 60, 1120-1139, https:// doi.org/10.1175/1520-0469(2003)060<1120:CMTAPP $>$ 2.0.CO;2.

Zhang, J. A., and F. D. Marks, 2015: Effects of horizontal diffusion on tropical cyclone intensity change and structure in idealized three-dimensional numerical simulations. Mon. Wea. Rev., 143, 3981-3995, https://doi.org/10.1175/MWR-D14-00341.1. 
_- R. F. Rogers, D. S. Nolan, and F. D. Marks, 2011: On the characteristic height scales of the hurricane boundary layer. Mon. Wea. Rev., 139, 2523-2535, https://doi.org/ 10.1175/MWR-D-10-05017.1.

S. G. Gopalakrishnan, F. D. Marks, R. F. Rogers, and V. Tallapragada, 2012: A developmental framework for improving hurricane model physical parameterizations using aircraft observations. Trop. Cyclone Res. Rev., 1, 419-429, https://doi.org/10.6057/2012TCRR04.01.
D. S. Nolan, R. F. Rogers, and V. Tallapragada, 2015: Evaluating the impact of improvements in the boundary layer parameterization on hurricane intensity and structure forecasts in HWRF. Mon. Wea. Rev., 143, 3136-3155, https:// doi.org/10.1175/MWR-D-14-00339.1.

R. F. Rogers, and V. Tallapragada, 2017: Impact of parameterized boundary layer structure on tropical cyclone rapid intensification forecasts in HWRF. Mon. Wea. Rev., 145, 1413-1426, https://doi.org/10.1175/MWR-D-16-0129.1. 